\title{
The Canis Major dwarf galaxy as the progenitor of the Monoceros tidal stream
}

\author{
D. Martínez-Delgado ${ }^{1} \dagger$, J. Peñarrubia ${ }^{2}$, D.I. Dinescu ${ }^{3}$, D.J. Butler ${ }^{2}$ \\ and H.W. Rix ${ }^{2}$ \\ ${ }^{1}$ Instituto de Astrofísica de Andalucía (CSIC), Spain. ${ }^{2}$ Max-Planck-Institut-fur Astronomie, \\ Germany. ${ }^{3}$ Astronomy Department, Yale University, USA.
}

\begin{abstract}
The Sloan Digital Sky Survey has recently discovered a coherent ring of stars at low galactic latitude that is believed to be the tidal stream of a merging dwarf galaxy in the Galactic plane (named the Monoceros tidal stream). The existence and location of the core of its progenitor galaxy is still controversial. The best candidate is the Canis Major dwarf galaxy, a distinct overdensity of red stars discovered in the 2MASS survey, but also interpreted as the signature of the Galactic warp viewed in projection. In this paper, we report a variety of new observational evidence that supports the notion that CMa is the remnant of a partially disrupted core of a dwarf satellite. The comparison of the orbit derived from our theoretical model for the parent galaxy of this ring-like structure with an accurate determination of CMa orbit leads to the conclusion that this satellite is the best candidate for the progenitor of the Monoceros tidal stream
\end{abstract}

Keywords. Galaxy:formation, Galaxy:structure, galaxies:dwarf

\section{Introduction}

The Sloan Digital Sky Survey has recently discovered a coherent ring-like structure at low galactic latitude spanning about 100 degree in the sky and surrounding the Galactic disk at Galactocentric distances from $\sim 15 \mathrm{kpc}$ to $\sim 20 \mathrm{kpc}$ (Newberg et al. 2002; Yanny et al. 2003). The tremendous observational and theoretical effort to understand its origin have proven that the structural characteristic and kinematics of this stellar ring are consistent with the properties expected for the tidal stream of a merging dwarf galaxy in the Galactic plane (named the Monoceros tidal stream). If this ring is a tidal tail feature, it must have had a parent galaxy, which may or may not be completely disrupted by now.

Unlike the Sagittarius tidal stream, the Monoceros stream has been detected prior to locating the main body of its progenitor galaxy. The best available candidate is the Canis Major (CMa) dwarf galaxy, a strong elliptically shaped stellar overdensity of red giant stars discovered by Martin et al. (2004) from an analysis of the 2MASS survey. As an alternative interpretation, Momany et al. (2004) suggested that this overdensity is only the signature of the Galactic warp. In this paper, we address the controversy on the origin of this stellar system and its relation with the Monoceros stream by answering the following questions: Can we constrain the position of the progenitor galaxy with Nbody simulations from the distribution and kinematics of Monoceros tidal debris? Is the CMa over-density the remmnat of the core of a dwarf galaxy? And if it is, is CMa the progenitor of the Monoceros tidal stream?

\section{$\dagger$ email: ddelgado@iaa.es}




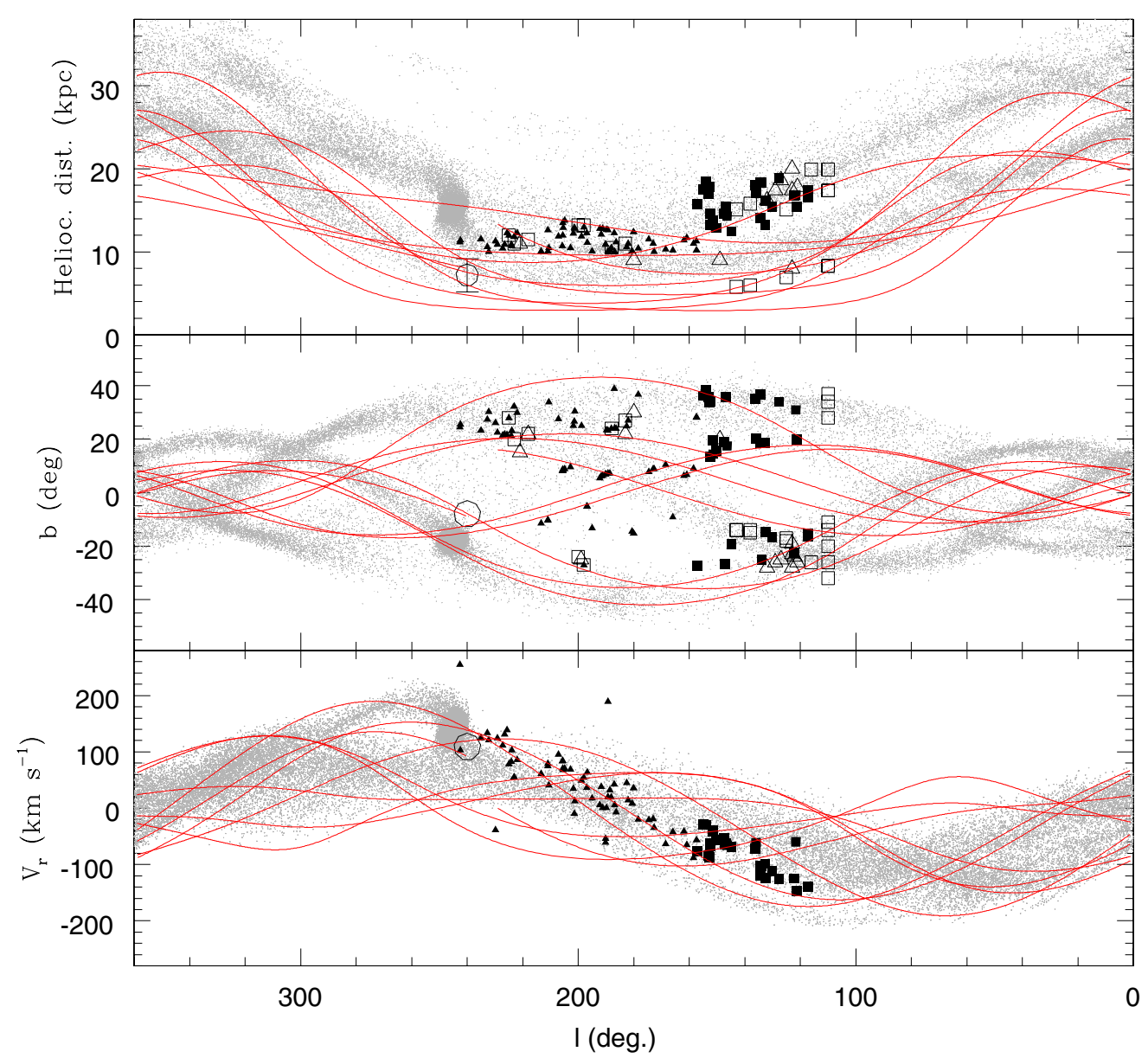

Figure 1. Comparison of the best fitting N-body simulation of the Monoceros tidal stream (small dots;see Peñarrubia et al. 2005 and references therein for a complete description of the Monoceros stream detections) with the orbit of the CMa dwarf derived from its absolute proper motion (solid line) by Dinescu et al. (2005)

\section{Constraining the progenitor position with theoretical model of the Monoceros tidal stream}

Peñarrubia et al. (2005) have attempted to determine the present position of the Monoceros stream progenitor through N-body simulations. These authors constrain the motion and mass of a possible progenitor by fitting the available Monoceros stream detections to thousands of orbits of dwarf galaxies with different masses. This method has been proven to provide powerful constrains on the eccentricity and orbital inclination of the possible progenitor, finding that the best solutions are for a satellite galaxy moving on a low eccentric $(\mathrm{e}=0.10 \pm 0.05)$, low orbital inclination $(\mathrm{i}=25 \pm 5 \mathrm{deg})$ prograde orbit (Figure 1). Owing to the small area of the sky where the Monoceros stream has been detected, the solutions are degenerated for some free parameters of the model: Namely, the axis-ratio of the Milky Way halo, the mass and the present location of the stream progenitor cannot be sufficiently constrained. Focusing on the last point, the model reproduces the geometrical and kinematical distribution of debris if the progenitor's remnants are 


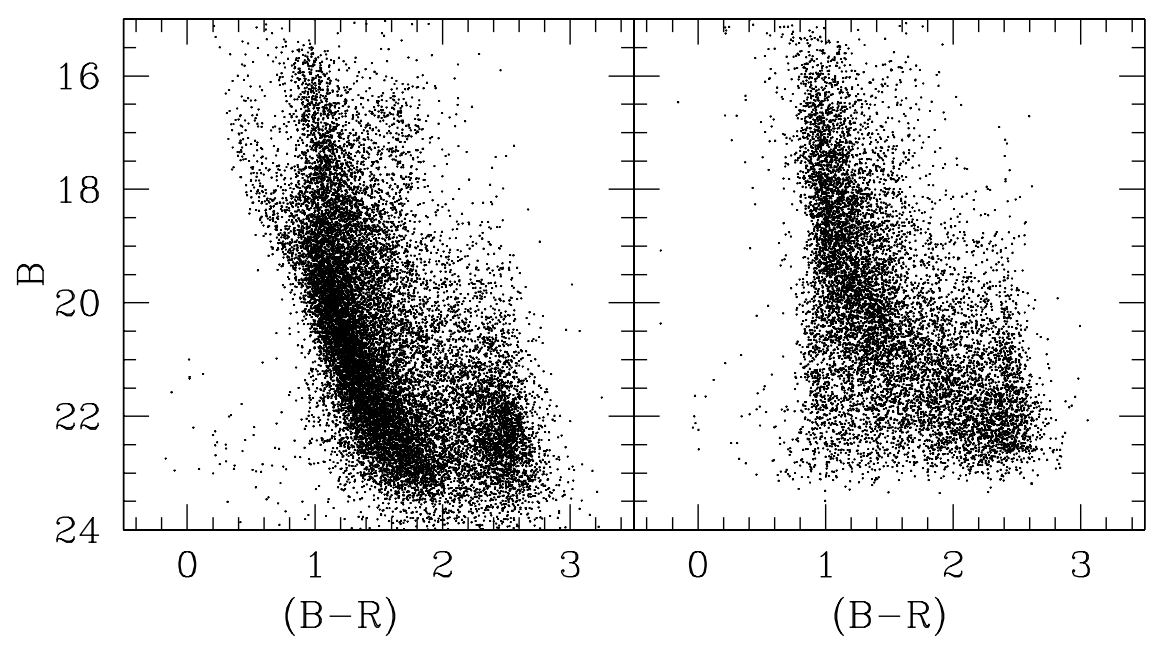

Figure 2. (left panel) Color-magnitude diagram of the center of the Canis Major overdensity $((l, b)=(240,-8))$ (see Martínez-Delgado et al. 2005 for a detailed description); (right panel) Color-magnitude diagram of a control field situated at $(1, b)=(240,+8)$, showing the distribution of Galactic background/foreground stars and an absence of the dwarf galaxy features.

located within $260>l>120$ at heliocentric distance of $\sim 14 \mathrm{kpc}$. That solution can be futher constrained with future detections in a wider range of longitudinal directions. The CMa system is located at $l=240$, which enters in the range obtained from N-body simulations, although at a closer distance $\left(d_{\text {helioc }} \sim 8 \mathrm{kpc}\right)$. The close distance of CMa appears to indicate that, if this system is the progenitor of the Monoceros stream, there are parts of the tidal stream at closer heliocentric distances that have not yet been detected.

\section{The nature of the Canis Major dwarf}

Fig 2a shows the color-magnitude diagram (CMD) of the putative center of the CMa over-density discovered by Martin et al. (2004). The most remarkable feature is a narrow main-sequence (MS), with a high constrast with respect to the thin/thick/halo background contamination, of which distribution is better observed in the CMD of a control field (Fig. 2b). The CMD morphology in Fig. 1a is in agreement with the expected for a stellar system composed by a mean metal-rich stellar population and that has undergone at least two distinct epochs of star formation (the last one only 1-2 Gyr ago).

From the MS feature, we derive a line-of sight depth for this system of $0.9 \pm 0.3 \mathrm{kpc}$, consistent with the interpretation of a remnant of a partially disrupted dwarf satellite (Martínez-Delgado et al. 2005). This limited line-of-sight depth is also very difficult to reconciled with the hypothesis of a warped Galactic outer disk viewed in projection, as proposed by Momany et al. (2004) (see also Sec. 3). The derived surface brightness $\left(\mu_{V, 0}=23.3 \pm 0.1 \mathrm{mag}\right)$ and absolute magnitude $\left(M_{V}=-14.5 \pm 0.1 \mathrm{mag}\right)$ of CMa places it in the category of dwarf galaxy in the known size-luminosity relation followed by dwarf galaxies (see Pasquali et al. 2005). Additional evidence on the dwarf nature of CMa from its orbital motion can be found in Sec. 4. At a distance of $8 \mathrm{kpc}, \mathrm{CMa}$ is the closest dwarf galaxy known. 


\section{The orbit of the Canis Major dwarf}

The question about whether the CMa dwarf could be the progenitor of the Monoceros tidal stream cannot be answered without a better constrain on the motion of this posible satellite. Recent absolute proper motion measurements of a sample of bona-fide CMa star members (Dinescu et al. 2005) provide an accurate determination of the CMa orbit. The orbit has a pericenter of $10 \pm 0.9 \mathrm{kpc}$ and an apocenter of $14 \pm 0.2 \mathrm{kpc}$. The orbit inclination is $15 \pm 3 \mathrm{deg}$ and the eccentricity is $0.14 \pm 0.04$. Currently, CMa is at its apocenter, and it should undergo tidal disruption.

The orbital motion of the CMa also provides important clues on the controversy about the origin of this stellar system (dwarf galaxy versus Galactic pertubation). While the CMa orbit is not very dissimilar from orbits of thick disk stars, the Monoceros stream stars reach a maximum distance from the Galactic plane of $\sim 2 \mathrm{kpc}$, that is larger than the thick disk scale height $(\leqslant 1 \mathrm{kpc})$. In addition, the derived $\mathrm{W}$ velocity component $(\mathrm{W}=-49 \pm 15 \mathrm{~km} / \mathrm{s})$ shows significant $(3 \sigma)$ motion perpendicular to the the disk and its negative value is inconsistent $(7 \sigma)$ with the expected motion (e.g., Drimmel, Smart \& Lattanzi 2000) of the warp at this Galactic location (see Dinescu et al. 2005).

The derived orbit parameters are fairly similar to those predicted in the theoretical model by Peñarrubia et al. (2005; see Sec. 3) for the Monoceros stream's progenitor (within $1 \sigma$ ). Fig. 1 shows a comparison between the best fitting N-body model (small dots) of the Monoceros stream and the orbit of CMa derived from its proper motion (solid line). This agreement supports the argument that $\mathrm{CMa}$ is the best candidate for the parent galaxy of the Monoceros stream.

\section{References}

Dinescu, D.I., Martínez-Delgado, D., Girard, T.M., Peñarrubia, J., Rix, H.W., Butler, D.J. \& van Altena, W.F. 2005, ApJ Letters submitted

Martin, N.F., Ibata, R.A., Bellazzini, M., Irwin, M.J., Lewis, G.F. \& Dehnen, W. 2004, MNRAS 348,12

Drimmel, R., Smart, R.L. \& Lattanzi, M.G. 2000, A 6 A 354,67

Martínez-Delgado, D., Butler, D.J., Rix, H.W., Franco, Y.I., Peñarrubia, J., Alfaro, E.J. \& Dinescu, D.I. 2005, ApJ (Letters) in press (astro-ph/0410611)

Momany, Y., Zaggia, S.R., Bonifacio, P., Piotto, G., De Anfeli, F., Bedin, L.R. \& Carraro, G. 2004, A\&3A 421, L29

Newberg, H.J. et al. 2002, ApJ 569, 245

Pasquali, A., Larsen, S., Ferreras, I., Gnedin, O.Y., Malhotra, S., Rhoads, J.E., Pirzkal, N. \& Walsh, J.R. 2005, AJ 129, 148

Peñarrubia, J., Martínez-Delgado, D., Rix, H.W. et al. 2005, ApJ (in press) (astro-ph/0410448)

Yanny,B. et al. 2003, ApJ 588, 824

\section{Discussion}

MATEO: a)Was the Galactic potential in the model static?; b) How long did the model run?; c)What is the total mass of the contents of the stream?

Martínez-Delgado: a) In our simulations, the Galaxy reacts to the presence of the Monoceros stream, but our Galaxy model does not implement a cosmological evolution; b) The available observational data can be reproduce within the last 3 Gyr of a possible progenitor orbit; c) The initial mass was $6 \times 10^{8} M_{\odot}$ and the satellite lost half of the mass at the end of the simulation. 